\title{
APPLICATION OF THE SOLUBILITY PARAMETER CONCEPT TO THE DESIGN OF CHEMIRESISTOR ARRAYS
}

\author{
R. C. Hughes, M. P. Eastman, * W. G. Yelton, A. J. Ricco, S. V. Patel, and M. W. Jenkins \\ Sandia National Laboratories, Albuquerque, New Mexico, 87185-1425 \\ *Department of Chemistry, Northern Arizona University, Flagstaff, Arizona, 86011
}

\begin{abstract}
Arrays of unheated chemically sensitive resistors (chemiresistors) can serve as extremely small, low-powerconsumption sensors with simple read-out electronics. Most work has focused on the exotic polymeric organic metals, but here we report new results on carbon-loaded polymer composites, as well as polymeric ionic conductors. We use the solubility parameter concept to understand and categorize the chemiresistor responses and, in particular, we compare chemiresistors fabricated from polyisobutylene (PIB) to results from PIB-coated acoustic wave sensors.
\end{abstract}

\section{INTRODUCTION}

Microsensor development for sensing vapors has often focused on creating single devices or arrays with the maximum selectivity to specific vapors [1-4]. Applications include systems for environmental monitoring, industrial process control, contraband detection, and the like. A problem having different constraints is the detection of solvent spills in areas containing electrical equipment: many different solvents may attack the insulators used in cables, connectors, wire bundles, and encapsulation, and the identity of the solvent is less important than its effect. Since the nature of this challenge depends on a range of unpredictable factors, good design dictates the development of a system using the minimum number of sensors capable of responding to all possible solvents. In addressing this problem, we sought to design a series of chemiresistors, whose responses span "all solvent space," that would be planar, extremely small, and compatible with silicon microelectronics. With today's miniaturized communications technology, it is possible to envisage extremely small packages that include the sensors, analog electronics, and telemetry for transmission of sensor data.

In discussing "solvent space," it is important to have at least a semi-quantitative measure of solvent-polymer interactions. The solubility parameter, $\delta$, introduced by Hildebrand [5], has been widely used as a tool to find the best solvent for a particular polymer. For a given solvent (or polymer), $\delta$ is loosely related to its cohesive energy and, thus, the energy of interaction among the molecules of the substance. Large tables compile measured solubility parameters for both solvents and polymers [6].

The majority of the results reported here are for carbon particle/insulating organic polymer composites. There is a long history of these composites being applied as conductive, flexible films; commercial products include self-regulating heating tape and resettable fuses, both of which exploit these materials' positive temperature coefficient of resistance (PTC) [7]. A number of papers have also reported the chemical sensing properties of these films $[2, \mathbf{8}, \mathbf{9}]$. The temperature, pressure, and chemical response of these films all depend on the same physics: the film resistance is a strong function of the volume fraction occupied by the conductive particles. If the polymer host increases its volume by thermal expansion or swelling due to absorption of analyte molecules, the resistance increases due to breakage of some of the conductive pathways through the film. The magnitude of the response of such chemical sensors appears to depend almost entirely on the solvent- polymer interactions, with the conductive particles serving to report the degree of swelling of the host polymer matrix.

We also examined a second class of chemiresistor material, those relying upon the mobility of ions in an ionically conductive matrix to report the presence of an analyte. It is well documented that the ionic conductivity of such films is enhanced by solvents, particularly water [2]. The increased mobility can result from reduced viscosity of the polymer matrix when plasticized by the analyte, dielectric screening of the charged polymer backbone by the analytes [10], or solvation of the mobile ions.

\section{EXPERIMENTAL DETAILS}

Planar interdigitated electrodes (IDEs), fabricated at Sandia, consist of quartz platforms $(5 \times 8 \mathrm{~mm})$ supporting 50 pairs of photolithographically defined interdigitated electrodes $\left(1.6 \mathrm{~mm}^{2}\right.$ overall) comprised of $2000 \AA$ of gold on a 150 - $\AA$ chrome adhesion layer (both metals thermally evaporated). The $1.6 \mathrm{~mm} \times 8$ $\mu \mathrm{m}$-wide electrodes are separated by $8 \mu \mathrm{m}$. A widely spaced ( 3 $\mathrm{mm}$ ) pair of 6-mm-long electrodes was also fabricated on the same chip, providing higher resistance for those materials having exceptionally low resistance across the IDEs.

Insulating polymer/conductive particle composites were prepared by co-dissolving a nonconductive organic polymer and $40 \%$-by-weight (relative to the polymer) of $30-\mathrm{nm}$ graphıtized carbon particles in an appropriate solvent, then coating the IDEs by spin-coating or deposition from a pipette. The polymer matrix materials include polyisobutylene (PIB); polydiphenoxyphosphazine (DPPZ); syndiotactic polybutadiene (PBS); polyvinylalcohol (PVA); and polyethylene/vinylacetate copolymer (PEVA). These were selected to cover a wide range of solubility parameters.

Two types of ionically conductive composites were prepared. An organic polymer/liquid crystal composite device was made by dispersing $0.3 \mathrm{~g}$ of the nematic liquid crystal "K21" in $0.24 \mathrm{~g}$ of PVA (MW $=25,000)$ dissolved in $2-3 \mathrm{ml}$ of water; 3 $\mathrm{mg}$ of tetraethylammonium bromide was added to provide mobile ions. A second material was based upon hectorite clay, which includes mobile cations intercalated between sheets of fixed anionic sites. A gold colloid/hectorite coating was prepared by suspending sodium hectorite (NL Industries, as-received) in a solution of colloidal gold (Unconjugated Polygold, $40 \mathrm{~nm}$; Polysciences, Inc.). Sensors were prepared by spin coating or smearing a thin film on the IDE. Impedance spectroscopy was used to characterize the response of the ionically conductive films: the optimal frequency range was found to be $2 \mathrm{~Hz}-1 \mathrm{kHz}$.

Analytes included isooctane, cyclohexane, toluene. trichloroethylene, xylene, DIMP (diisopropylmethylphosphonate), ethanol. methanol, and water. Solvents and analytes were commercially available (Fisher Chemical) and used as received, except ethanol, which was distilled to remove water.

IDE devices were placed in a stainless-steel fixture housed in a constant-temperature oven. Nitrogen gas from a cryogenic source was passed through gas bubblers filled with the analyte and maintained at room temperature. Flow rates were controlled by mass-flow controllers. Resistance measurements were made using a Keithley Model 2001 Multimeter or, in the case of highimpedance ( $>10^{7} \mathrm{ohms}$ ) devices requiring AC measurement at 


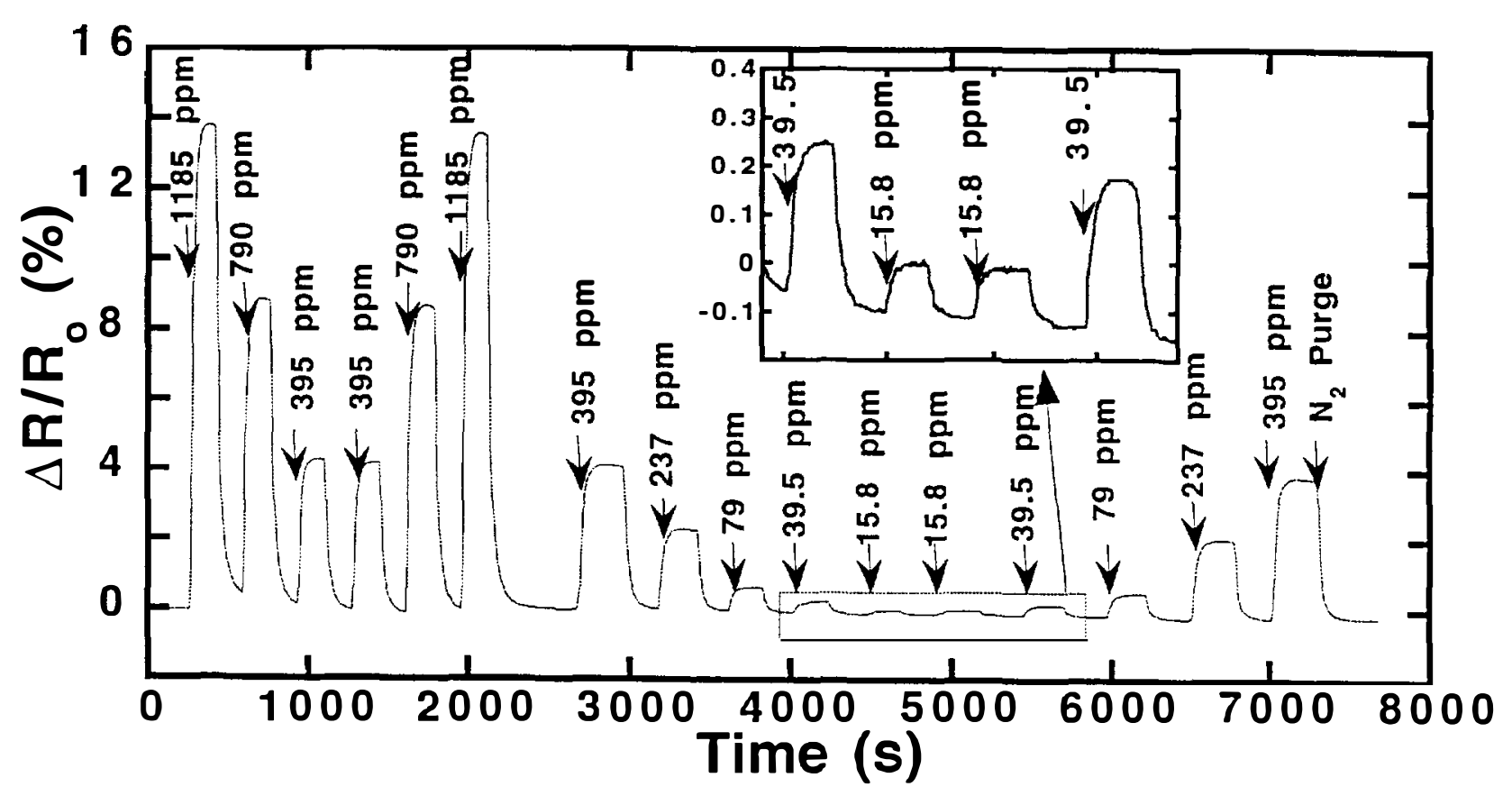

Figure 1. Response of a chemiresistor to pulses of different concentrations of m-xylene in $\mathrm{N}_{2}$. The sensor is a carbon particle-loaded $\left(40 \%\right.$ by weight) film of $P I B$ held at $32^{\circ} \mathrm{C}$. The inset expands the response at low partial pressures of xylene.

specific frequencies, a Stanford Research Systems SR 830 DSP Lock-In Amplifier interfaced to a Keithley Model 427 current amplifier. LabVIEW was used to set flow controllers and acquire data from the multimeters.

\section{RESULTS AND DISCUSSION}

We studied two important polymers not previously reported in the literature of carbon/polymer composite sensors. The first of these. PIB, is a rubbery polymer that has been utilized extensively as a chemically sensitive coating on surface acoustic wave devices $[3,11]$. As a consequence, there is a large body of information on the interaction of organic vapors with thin films of this polymer, including the isotherms (sensor response vs. vapor partial pressure), speed of response for both absorption and desorption, and response to mixtures of vapors.

Figure 1 shows the response of a carbon-loaded PIB film to pulses of various concentrations of $m$-xylene vapor.The sensor temperature was maintained at $32^{\circ} \mathrm{C}$ in the oven; the bubbler temperature was $23^{\circ} \mathrm{C}$. The equilibrium vapor pressure of $m$-xylene at $21^{\circ} \mathrm{C}$ is $7900 \mathrm{ppm}$. The partial pressures in the pulses of vapor are given in Figure 1. Response and recovery are rapid, and reproducibility is good. The inset in Figure 1 expands the responses at the lowest partial pressures.

The responses in Figure 1 are similar to those reported for PIB films on acoustic wave devices, consistent with the notion that the concentration of analyte in the film determines both the magnitude of the acoustic wave device response. and the swelling of the polymer matrix, which in turn affects the volume fraction occupied by the carbon particles and hence film resistance.

The PIB chemiresistor responds to a variety of organic vapors. Figure 2 shows the relative response to seven different solvents as a function of solubility parameter. Plotted for comparison are the relative responses of a PIB-coated acoustic wave device [11]. The PIB has a $\delta$ value of $15.5[6]$, and the peak responses of both the chemiresistor and SAW data are nearby, at $17-18$. Obtaining a large sensor response for matching $\delta$ values holds, but not with high accuracy.

The second important polymer system is PVA. This polymer is readily prepared as a thin film and is expected to interact especially strongly with polar molecules $(\delta=25.8$ [6]); as a consequence, it should respond to analytes that might not produce a strong response in a PIB-based sensor. We have incorporated PVA into our sensors in two distinct ways. The first is in the form of the carbon-loaded polymer composite: the second is in the form of an ionically conductive mixture of PVA-dispersed liquid crystal, doped with an organic salt to confer conductivity.

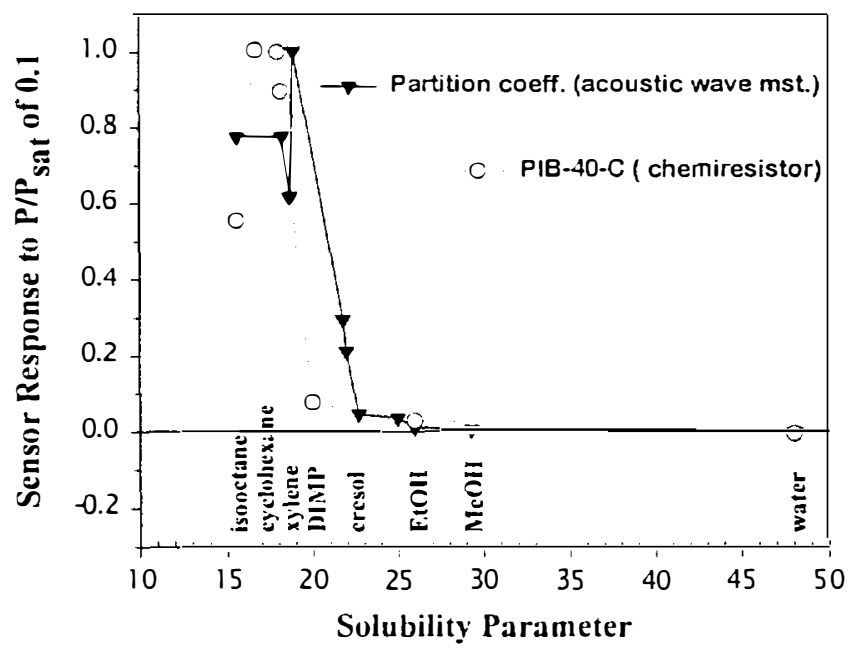

Figure 2. Normalized response of the PIB chemiresistor to a variety of solvents with the maximum signal at $10 \% P / P_{\text {sat }}$ set to 1 . These data are compared with the SAW-measured partition coefficients (6) for the same solvents (plus some additional ones) and plotted vs. the solubility parameters of the solvents. A number of the solvents are labeled on the figure. This suggests that the chemiresistor response is closely related to the swelling of the polvmer phase.

The PVA/liquid crystal system is reminiscent of the multicomponent polymer electrolyte sensors recently reported by Cammann et al. $\mid 12\}$; however. it is important to recognize that the 
liquid crystal imparts some important properties to the sensor. First, the liquid crystal segregates into microdroplets as the film forms on the sensor; thus, the films are two-phase materials, and each phase can respond differently to various vapors. Thus, the potential exists for polymer-dispersed microdroplet materials to respond to a broad range of analytes and concentrations.

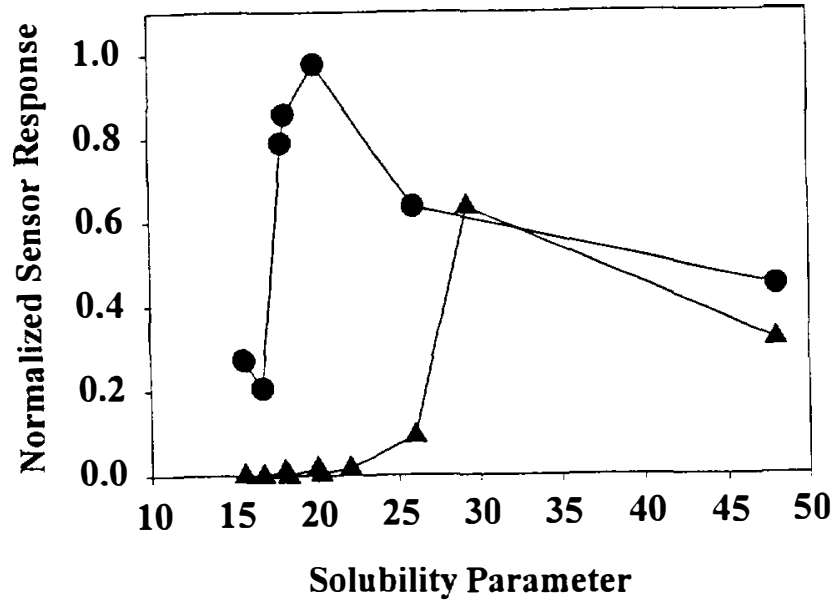

Figure 3. Normalized response of the two types of PVA-based sensors, plotted vs. solubility parameter as in Figure 2. The twophase $P V A / K 21$ has a broader response than the single-phase $P V A$, but requires $A C$ voltages for measurement.

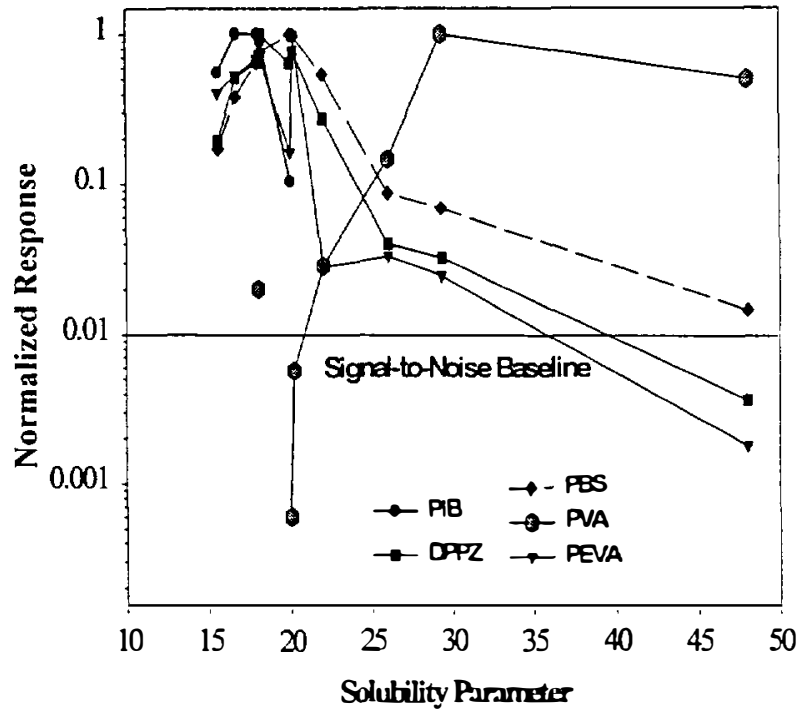

Figure 4. Responses of five carbon/polymer composite sensors vs. solvent solubility parameter. The log scale helps to separate the responses; the "Signal-to-Noise Baseline" gives an indication of the size of signal below which data are generally unreliable due to drift and noise. The symbols identify the polymers, which are defined in the Experimental section.

Figure 3 shows the response curves for PVA/K21 and carbon-loaded PVA vs. solubility parameter. The PVA/carbon film shows a peak response for methanol $(\delta=29.3)$ and good response to relative humidity; it responds weakly at lower $\delta$ values. In contrast. the two-phase PVA/K21 responds at both high and low $\delta$ values. The responses for PVA/K2I were measured at $2 \mathrm{~Hz}$ and $0.5 \mathrm{~V}$ using the lock-in amplifier setup. Impedance $v s$. frequency curves were similar to poly(hexane viologen) responses we reported elsewhere [10].

Figure 4 uses the format of Figs. 2 and 3 to plot the responses of three other carbon/polymer composites: DPPZ, PEVA, and PBS, plus PVA. There are some unpredicted features in these responses, such as the low response for DIMP compared with trichloroethylene for PEVA, but no solvent goes unmeasured by at least one of the sensors.

\section{PATTERN RECOGNITION}

Having an array of sensors not only provides responses to a wide variety of solvents, it also presents an opportunity to identify particular solvents using pattern-recognition (PR) techniques. VERI PR, developed at Sandia, has been used extensively by us to interpret data from acoustic wave and catalytic gate sensors [4, 13]. Application of VERI PR is illustrated in Figure 5, showing the responses of three chemiresistors to four solvents in a 3-D plot. Each point represents a different concentration of the indicated solvent. The responses have been equalized and normalized so that each response is a vector ending on the surface of a unit sphere (outlined by the solid curves ). The readers' vision allows her/him to see that classification of the signals from each of the four solvents is unambiguous at all tested concentrations. VERI PR can be used for large arrays (higher dimensions than three); it reports whether the response to an unknown sample clusters with any previously tested classes, or must be treated as a previously untested chemical.

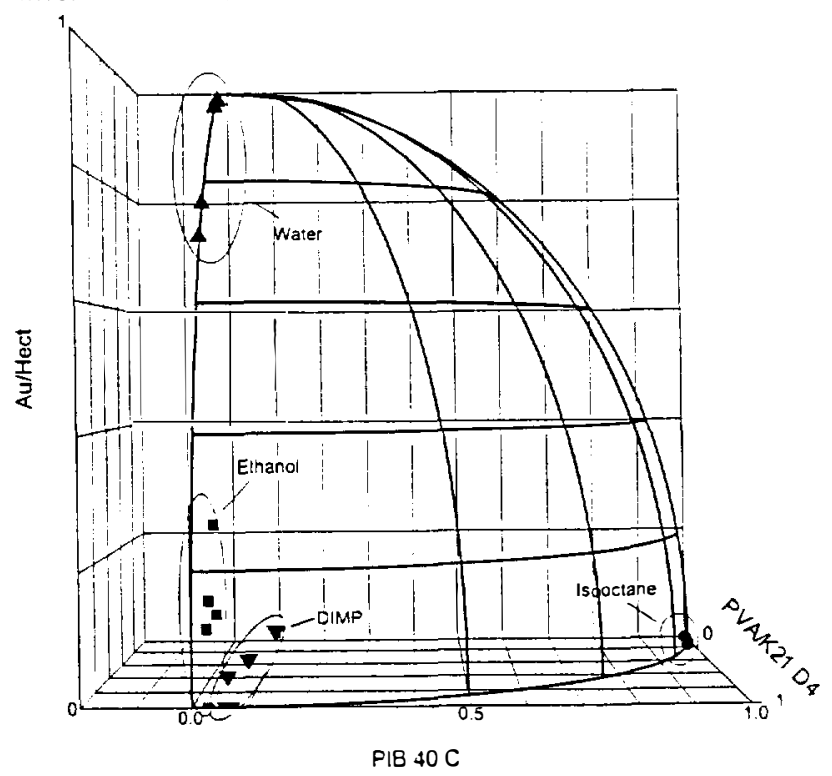

Figure 5. Pattern recognition plot to identify individual solvents from the response to three sensors. Each point represents a different concentration of the labeled solvent; each axis represents the normalized response of one of the sensors.

\section{THE CHEMICAL FUSE}

An interesting and valuable property of the carbon-loaded polymer/composite chemiresistors is that very high concentrations of solvent cause orders-of-magnitude increase in their resistance. In cases where the polymer can completely dissolve in the solvent. and with sufficient mechanical agitation, the sensor will permanently remain at high (or infinite) resistance; the sensor is destroyed. In some cases. however, the solvent will eventually evaporate in much the same way that the sensor was originally fabricated, restoring the sensor.

An example is given in Figure 6. where a drop of liquid isouctane was placed on a horizontal PIB-based sensor. The sampling period was $100 \mathrm{~ms}$; in less than one second, the resistance 
increased over three orders of magnitude. As long as liquid isooctane remained visible on the sensor, the resistance remained very high ( $300 \mathrm{M} \Omega$ ), but measurable. As the isooctane evaporated, a sharp drop in resistance was observed. The sensor was still functional for sensing lower concentrations, but the baseline resistance had decreased permanently. Subsequent doses of liquid isooctane always produced large increases in resistance, but the "under-liquid" and baseline resistances changed by smaller amounts. Further engineering is required to make a reproducible fuse similar to the commercially available PTC electrical fuse [7].

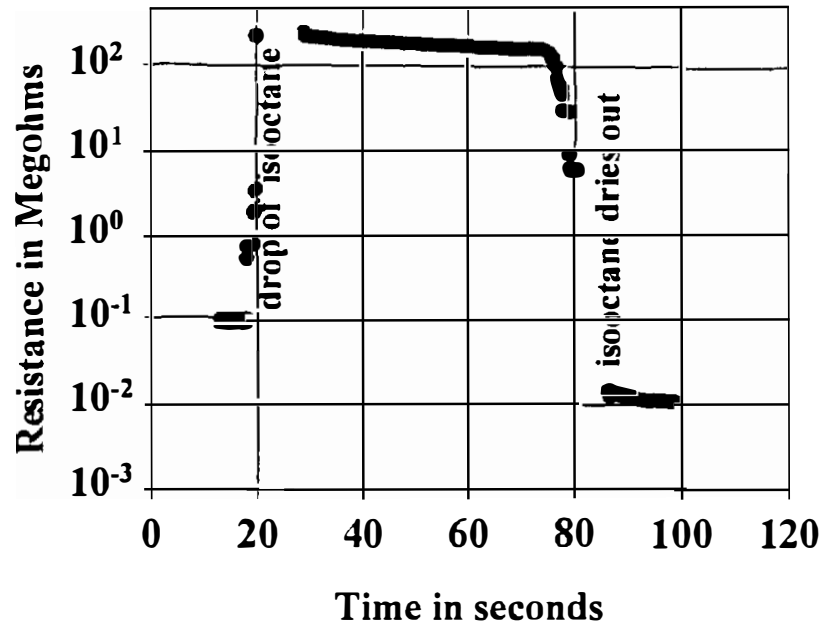

Figure 6. Resistance of a PIB sensor acting as a (resettable) chemical fuse to liquid isooctane. A rapid increase in resistance results from a small drop of isooctane $(0.1$ seconds per data point from a 16-bit digitizer) and recovery occurs when the isooctane evaporates.

\section{SUMMARY AND CONCLUSIONS}

We believe the data in this paper demonstrate that an extremely small, low-power-consumption array of chemiresistors can identify almost any kind of solvent spill. This preliminary report builds the framework for further efforts to develop the "universal solvent sensor."

Solubility parameters were used to characterize sensor films and as an aid in selecting sensors for use in arrays to discriminate between members of similar or dissimilar solvent classes. Chemiresistors based on several carbon-loaded polymers were studied, along with an ionically conductive, polymer-dispersed liquid crystal system. The various sensors show differing sensitivities in different regions of "solvent space." PIB shows maximum sensitivity to analytes characterized by small $\delta$ values, while PVA responds best to solvents with large $\delta$ values. The PVA/K21 two-phase film has the broadest response range of the materials evaluated. Additional data must be acquired to provide adequate training sets for determining a range of unknowns in the field.

Figure 4 illustrates the feasibility of selecting 3 or 4 sensors. at least one of which responds to every possible solvent above some threshold concentration. Although some solvent might produce too small a response to be detected, our logic in protecting electronic components and packaging is to select polymers representative of those used in such applications. Figure 6 shows how chemiresistors can act as resettable chemical fuses to give rapid, unambiguous warning of a solvent spill. The chemical fuse failure mode is high resistance, so other hazards such as fire, physical destruction, or strong oxidizing agents (e.g., nitric acid) will yield a similar failure response.
Conductive particle/polymer composite sensors can be fabricated in an extremely small $\left(<1 \mathrm{~mm}^{2}\right)$ planar format and consume almost no power to measure DC resistance. Sensor arrays are readily fabricated using fluid-dispensing techniques. Ionic conductivity-based sensors are equally small, but require more complex circuitry for AC measurement; unique selectivity or sensitivity can make these materials attractive nonetheless.

\section{ACKNOWLEDGMENTS}

Sandia is a multiprogram laboratory operated by Sandia Corporation, a Lockheed Martin Company, for the United States Department of Energy under Contract DE-AC04-94AL85000. MPE acknowledges sabbatical support by Northern Arizona University and supplementary financial support by Sandia National Laboratories.

\section{REFERENCES}

1. Techniques and Mechanisms in Gas Sensing, P. T. Moseley, J. O. W. Norris, and D. E. Williams, Eds., IOP Publishing (Adam Hilger Series on Sensors), Bristol, England (1991).

2. G. Harsanyi, Polymer Films in Sensor Applications, Technomic Publishing, Lancaster (1995).

3. D. S. Ballantine, Jr., et al. Acoustic Wave Sensors: Theory, Design and Physico-Chemical Applications, Academic Press, Inc., San Diego (1997), Ch. 5.

4. A. J. Ricco, R. M. Crooks, and G. C. Osbourn, "SAW Chemical Sensor Arrays: New Chemically Sensitive Interfaces Combined with Novel Cluster Analysis to Detect Volatile Organic Compounds and Mixtures," Accts. Chem. Res., 31, xxxx (1998).

5. D. W. Van Krevelen, Properties of Polymers, Elsevier. New York (1976), Ch. 7.

6. E. A. Grulke, in Polymer Handbook, 3rd Edition, J. Brandrup and E. H. Immergut. Eds., Wiley and Sons, New York, (1989), p. VII/519.

7. M. B. Heaney, "Resistance-Expansion Temperature Behavior of a Disordered Conductor-Insulator Composite," Appl. Phys. Lett., 69, 2602 (1996).

8. M. C. Lonergan, E. J. Severin, B. J. Doleman, S. A. Beaber, R. H. Grubbs, and N. S. Lewis, "Array-Based Vapor Sensing using Chemically Sensitive Carbon Black-Polymer Resistors," Chem. Mater., 8, 2298 (1996).

9. B. Lundberg and B. Sundqvist, "Resistivity of a Composite Conducting Polymer as a Function of Temperature. Pressure and Environment: Applications as a Pressure and Gas Concentration Transducer," J. Appl. Phys., 60, 1074 (1986).

10. K. B. Pfeifer, R. C. Hughes, M. W. Jenkins, and T. W. Schneider, "Viologen Polymer-Coated Impedance Sensors for Midrange Moisture Sensing," J. Electrochem. Soc., submitted for publication.

11. R. A. McGill, M. H. Abraham, and J. W. Grate. "Choosing Polymer Coatings for Chemical Sensors," Chemtech. Sept. (1994), p. 27.

12. K. Cammann, K. Buhlmann, B. Schlatt, H. Muller, and A. Choulga, "Multicomponent Polymer Electrolytes: New Extremely Versatile Receptor Materials for Gas Sensor and Electronic Noses," Technical Digest, Transducers '9?. IEEE, New York (1997), p. 1395.

13. G. C. Osbourn, J. W. Bartholomew, G. C. Frye, and A. J. Ricco, "Clustering-Based Pattern Recognition Applied to Chemical Recognition Using SAW Array Signals", Technical Digest, Solid-State Sensor and Actuator Workshop. Transducers Research Foundation. Cleveland (1994), p. 193. 